\title{
ARBOLARIA: EL ESTATUTO DE LA MACHI EN CAUSAS JUDICIALES POR MALEFICIO EN CHILE COLONIAL (s. XVII- XVIII)
}

Arbolaria: The status of the machi in maleficio trials in colonial Chile (XVII-XVIII)

\author{
Eduardo Valenzuela Avaca*
}

\section{RESUMEN}

La lucha contra los cultos locales en América fijó tempranamente la necesidad de controlar la influencia de los ministros de idolatrías o ministros demoníacos, agentes articuladores de la ritualidad indígena encargadas de hilvanar el tejido simbólico de las comunidades. Para el caso chileno, las machi ocuparon un lugar central en las disposiciones sinodales, como reflejo -a menor escala- de esta operatoria general, siendo castigada la mera asociación con estas figuras contaminantes. Sin embargo, en el contexto de las causas por maleficio llevadas adelante por la justicia ordinaria en el Chile del siglo XVIII su estatuto varía, contraviniendo no solo las reglas puestas por la justicia eclesiástica, sino también los propios modelos con los que la justicia ordinaria operó para enfrentar el ejercicio de las malas artes en Chile colonial. En este trabajo nos proponemos indagar sobre las causas de esta diferencia.

Palabras clave: Machi, maleficio, brujería, hechicería, mapuche.

\begin{abstract}
The fight against local cults in America set the need to control the influence of the ministros de idolatrias or ministros demoniacos, coordinating agents of the indigenous rituality responsible of basting the symbolic weave of the communities. In the Chilean case, the machi occupied
\end{abstract}

\footnotetext{
* Universidad de Chile, Chile / École des Hautes Études en Sciences Sociales, Francia. Correo electrónico: ejvalena@uc.cl
}

Artículo recibido el 19 de noviembre de 2014. Aceptado el 31 de julio de 2015. 
a central place in the Synod provisions, as a reflection -on a smaller scale- of the overall operative, being punished the mere association with these pollutants figures. However, in the context of the maleficio causes carried out by the Justicia Ordinaria in eighteenth century Chile, its status varies, not only contravening the rules set by ecclesiastical justice, but the own models used by the ordinary courts to face the practice of the malas artes in colonial Chile. In this article we intend to inquiry on the causes of this difference.

Keywords: Machi, maleficio, witcraft, sorcery, mapuche.

Los crímenes por maleficio -y por extensión, todas las formas de la superstitio - fueron, sin duda, algunos de los delitos de mayor dificultad probatoria tanto en la Europa medieval como en la colonia hispanoamericana. Los límites entre una práctica piadosa y otra ilícita eran difusos para la mayoría de la población, y de difícil solución teórica para los tratadistas, como es posible apreciar en la inagotable discusión del núcleo intelectual de la Iglesia frente a estos fenómenos. Estas reflexiones, inauguradas en la Península Ibérica con el hito fundacional del Concilio de Elvira en 324, se extendieron a lo largo de toda la Edad Media en un lento tránsito desde las prácticas supersticiosas de los rustici e illiterati hasta la consolidación del modelo de la brujería tardo-medieval, siempre adaptando su instrumental teórico a los nuevos enemigos de la cristiandad ${ }^{1}$.

No existieron nuevos modelos para el nuevo mundo. El control del maleficio y la superstición en América se nutrió de matrices preexistentes, unas en plena vigencia y operatividad, otras cuya utilidad había cesado para el contexto europeo, pero que podían volver a utilizarse si el caso así lo ameritaba. Las leyes indianas hicieron escasas modificaciones al corpus procedimental sentado en las Siete Partidas, código legal compuesto durante el gobierno de Alfonso X en el siglo XIII, y cuyo contexto de heterodoxia fue sensiblemente distinto al de la hispanoamérica colonial $^{2}$. La extirpación idolátrica, por ejemplo, una problemática fundamental

\footnotetext{
${ }^{1}$ Sobre el general del conflicto Cristianismo-Paganismo en la España temprana, ver: Serrano Sanz, Rosa. "Hacia un nuevo planteamiento del conflicto paganismo-cristianismo en la Península ibérica". Revista 'Ilu, Madrid, 1995. Para una revisión del problema de la superstición y la idolatría a la luz de la documentación conciliar, ver: Valenzuela, Eduardo: Vino nuevo en odres viejos: Superstitio et idololatria en la España alto-medieval. En José Manuel Cerda (Ed.), El mundo medieval: Legado y Alteridad. Santiago de Chile: Editorial Finis Terrae, 2009. Una revisión general del fenómeno heterodoxo en la Alta Edad Media en: Giordano, Oronzo. Religiosidad popular en la Alta Edad Media. Madrid: Gredos, 1983. Para un seguimiento de la constitución de un modelo condenatorio tardo-medieval, Ginzburg, Carlo. Historia Nocturna, un desciframiento del aquelarre. Barcelona: Munchkin Editores, 1991.

${ }^{2}$ Dougnac, Antonio. "El delito de hechicería en Chile Indiano". Revista Chilena de Historia del Derecho (8): 93-107, (1981: 98). La influencia de las Siete Partidas en el derecho indiano constituye un tema
} 
tanto en el ordenamiento del mundo indígena americano como para la Hispania temprana, no constituía un problema para la Iglesia de la Europa del siglo XIII, preocupada fundamentalmente por las viejas supersticiones y vanas observancias, así como por el ascendente protagonismo de las herejías que desembocarán en las persecuciones de los siglos posteriores.

A diferencia de los tribunales eclesiásticos, la justicia real -en abstractoo los jueces locales -como figuras específicas- no poseían el nivel de formación de su contraparte eclesiástica. La nutrida producción de los tratadistas teóricos, el acceso al corpus casuístico de la tradición conciliar, la formación profunda en los distintos aspectos del dogma, son solo algunos de los atributos que evidencia la posición aventajada que los tribunales eclesiásticos tenían por sobre los jueces de la justicia ordinaria a la hora de fallar sobre crímenes de fuero mixto. Nada de esto impidió, por cierto, que estos últimos pudiesen operar sobre un conjunto de modelos y matrices construidas en base a la diseminación natural de muchas de estas informaciones ${ }^{3}$.

Los intereses eran, sin embargo, notoriamente distintos. Concentrados en el aspecto exterior del delito, los jueces locales se aproximaron al maleficio como una suerte de homicidio por vía de arte maxico, es decir, a través de un saber capaz de mutar sustancias, gestos y voces inocuas, en efectos maravillosos que excedían su condición natural ${ }^{4}$. Aunque conceptualmente constituía un delito de "fuero mixto", el peso de su efecto "externo" - la enfermedad o muerte de un miembro de la comunidad-determinó su jurisdicción a los tribunales ordinarios.

ampliamente documentado. No solo fue la fuente de derecho más consultada por las audiencias de las Indias, sino que su aplicación fue aun más extensiva y efectiva en las Indias que en la península misma, donde debía enfrentar la resistencia de cuerpos legales aun en vigencia. Para una revisión sobre su influencia, ver, entre otros: Ots y Capdequi, José María. Manual de historia del derecho español en las indias y del derecho propiamente indiano. Buenos Aires: Losada, (1945): 85-88. García Gallo, Alfonso. "Metodología de la historia del derecho indiano". Santiago de Chile: Publicaciones del Seminario de Historia y Filosofía del Derecho de la Facultad de Ciencias Jurídicas y Sociales de la Universidad de Chile, (1970): 73. Pérez González, María Luisa. "Los caminos reales de América en la legislación y en la historia". Anuario de Estudios Americanos, 58/1, (2001): 42.

${ }^{3}$ Para una identificación y problematización de estos modelos, ver: Valenzuela, Eduardo. Maleficio. Historias de hechicería y brujería en Chile colonial. Santiago de Chile: Pehuén Editores, 2013. Todos los fundamentos teóricos sobre los que se sostiene esta investigación están contenidos en el mismo estudio citado.

${ }^{4}$ La sencilla, pero precisa, definición del Diccionario de Autoridades sintetiza en gran medida la carga del término y el uso que aquí le daremos "Magia: ciencia o arte que enseña a hacer cosas extraordinarias y admirables". Diccionario de la lengua castellana en que se explica el verdadero sentido de las voces, su naturaleza y calidad con las phrases o modos de hablar, los proverbios o refranes, y otras cosas convenientes al uso de la lengua. Madrid: En la Impr. de Francisco del Hierro, 1726-1739. 6 v.(Edic. Facsimilar). fol. 455. El Diccionario de Covarrubias (1611), por su parte, no registra la entrada "Magia". 
Las causas judiciales por maleficio que constituyen el corpus de nuestro trabajo, fueron organizados y conducidos a partir de dos esquemas fuertemente estereotipados: hechicería y brujería. El primero, expresado como un saber personal, susceptible de ser enseńado, sustentado en la confección de artefactos mágicos (objetos "hechizos"), y sin adoración - latría - explícita al diablo o a la corporización de un demonio menor. El segundo, de carácter colectivo, articulado en función de intereses comunitarios, se desarrolló fuera de las ciudades centrales, en torno a espacios específicos como las cuevas (renü) de la tierra dentro. A diferencia de la hechicería, esta segunda práctica era asociada a formas de adoración explícita de entidades demoníacas, las cuales se manifestaban físicamente en las juntas secretas celebradas por la comunidad 5 . Los procesos que expondremos corresponden a estas dos estructuras organizadoras, siendo el maleficio el denominador común de ambas.

A la luz de estos modelos, resulta evidente la peligrosa posición en la que se encuentra la machi frente al actuar de la justicia. Todos sus recursos materiales, su conocimiento detallado de las propiedades visibles e invisibles de las plantas, su habilidad para identificar las potencias inherentes a algunos animales, y su capacidad para articular estas sustancias y crear nuevos dispositivos, constituyen rasgos inequívocos del ejercicio de la hechicería ${ }^{6}$ Si consideramos además su rol de mediación entre la comunidad y las entidades sagradas del mundo a partir de expresiones rituales con un distinto sello latréutico, la posibilidad de ser implicada en una causa por brujería resultaba evidente. Peor aún, la machi constituía una figura individualizada, un especialista con un rol que jugar en el sistema de creencias indígenas. El Sínodo Diocesano de 1626 dedica una de sus constituciones a esta problemática:

Item ordenamos y mandamos que en delante no permitan los que tienen cuidado de las almas de los indios que [...] llamaren machis para curarse con ellas por medio de invenciones del demonio o con hierbas que ofrecen al demonio con señales o gestos supersticiosos, los punimos y castigamos con la dicha excomunión

\footnotetext{
${ }^{5}$ Para las causas que nos convocan, las manifestaciones demoníacas incluían dos entidades que operaban como porteros de las cuevas: El Macho Cabrío o Chivato y una serpiente de grandes dimensiones denominada ynguayvilu. Ocasionalmente, la figura del Chivato podía ser reemplazada por una tercera entidad, el Ibunche. Un desarrollo de estos temas en: Valenzuela, Eduardo. Maleficio. Historias de hechicería y brujería en Chile colonial. Santiago de Chile: Pehuén Editores, (2013): 119. ${ }^{6}$ Una de las asociaciones más tempranas entre las machi y la hechicería la encontramos en los Sermones de Luis de Valdivia, donde se señala que los "malos indios" suelen "tomar consejo con los Machis (que son hechizeros) y con los viejos". Valdivia, Luis de: Sermón en lengua de Chile. De los mysterios de nuestra santa fe catholica, para predicarla a los indios infieles del reyno de Chile, dividido en nueve partes pequeñas, acomodadas a su capacidad. Santiago de Chile: Elzeviriana, 1897. Sermon II, Tit.13 (p. 11).
} 
mayor dicha en la constitución antecedentes, y a los indios o indias, negros o negras que hicieren las cosas sobredichas así mismo sean castigados con las penas puestas en la primera constitución ${ }^{7}$.

Hay algunos aspectos que son de nuestro interés en este anatema. En primer lugar, podemos observar que en las disposiciones sinodales, el ejercicio del machi se circunscribe a la sanación, y no se le considera sacerdote o ministro demoníaco, cargos que serán imputados a otras dos figuras ligadas a los ritos: Nancanes y Neges ${ }^{8}$. En segundo lugar, implícitamente se reconoce una doble naturaleza en la curación, ya como “invenciones del demonio", es decir, gestualidades y aspectos físicos del rito supersticioso que de acuerdo al pensamiento teológico no pueden tener potencia en sí mismos sino solo por intervención de potencias demoníacas; o por medio del uso de "hierbas", las cuales pese a poseer propiedades benéficas intrínsecas son ponderadas por la Iglesia como componentes de un rito con

\footnotetext{
7 "Sínodo Diocesano de Santiago de Chile celebrado en 1626, por el ilustrísimo señor Francisco González de Salcedo". Constitución Tercera. Transcripción y notas por Oviedo Cavada, Carlos, En: Revista Historia, (3): 313-360, Santiago de Chile, 1964. Las penas a las que hace referencia la constitución señala: "por la primera vez que esto hicieren les manden estar de rodillas un domingo con una soga a la garganta en presencia de los demás que acudieren a misa todo el tiempo que durare, y se les de una gran reprensión; exhortando a todos los demás que acudieren a misa a que se aparten de tales pecados y delitos. Y por la segunda vez les mandaran dar veinte o treinta azotes, despues de acabada la misa, habiendo estado como dicho es en presencia de los demás de rodillas. Y si perserveraren en su delito y les cometieren otra vez, nos lo remitan para que le mandemos castigar...". Constitución primera, Ibidem.

${ }^{8}$ Aunque introducirnos en estas figuras excede el propósito de este artículo, bien podemos señalar un par de antecedentes de ellas. La voz "nege" aparece en las Cartas Anuas de 1609-1614, específicamente en el contexto de los caciques de Elicura. Los misioneros recuerdan la llegada de un grupo de mensajeros de paz, a los cuales describen así: "los quinze delanteros traian en la mano un ramo de arbol de Canela en señal de paz, y los tres primeros le traian mucho maior, estos quinze venian entraje de Neges, que son a modo sacerdotes suyos con Vonetes Redondos e las cauezas y encima del vestydo unas yerbas delamar que llaman cochayuyos colgando muchos por delante y por detrás, amanera deberlas de dalmaticas, las quales son ynsignia entre ellos de una superstición [...] la qual solamente usan este tipo de paz y quietud" Documentos para la historia argentina. Tomo XIX. Iglesia. Cartas anuas de la provincia del Paraguay, Chile y Tucumán, de la Compañía de Jesús 1609 - 1614. Con advertencia de Emilio Ravignani e introducción del P. Carlos Leonhardt, Buenos Aires, Instituto de Investigaciones Históricas, Facultad de Filosofía y Letras, Peuser, 1927.PP. 235. La voz "nancan", por su parte, la encontramos en un proceso de brujería contra indígenas, en concepción, de la boca de una machi llamada Guenteray, de la que hablaremos más adelante. Señala: "[...] Panguicheo levanto la mata y la aparto a un lado de la cueba y quedo avierta y entraron por delante los Nancanes que son como ministros o sacerdotes de aquel sacrificio y que son cinco que el de maior estimasion es Guimiray yndia machi". Manuscritos Medina, T. 323, fojas 1-202. Sala Medina, rollo Ms. M85, Biblioteca Nacional, bajo el título: "Información levantada por el Capitán Don Antonio de Soto Pedreros, por orden del Presidente Don Tomás Marin de Poveda, contra varios indios acusados de brujos y hechiceros". Foja 47. De acuerdo a la testificación de Guenteray, no sería contraproducente el ejercicio de machi y el de nancan en un mismo sujeto.
} 
aspectos de falsa adoración. El Sínodo de Concepción 1744 recoge también la asociación del machi con la sanación, explicando su proliferación a partir de la ausencia de médicos, asumiendo siempre que su sanación está intrínsecamente ligada a prácticas demonológicas?.

¿Qué ocurre entonces con la machi en un contexto evidentemente dañino, como son las causas por maleficio sostenidas por la justicia colonial? ¿Cómo es ponderada una figura central de la ritualidad mapuche, como la Machi, en el contexto de un proceso por brujería? Aunque podríamos sentirnos tentados a extrapolar las disposiciones eclesiásticas a la esfera de la justicia ordinaria, hay antecedentes que nos llevan en otra dirección.

En septiembre de 1694 el capitán Antonio de Soto Pedreros, por orden de Tomás Marín de Póveda, es llamado a visitar las parcialidades de Imperial y Boroa por noticias sobre el ejercicio de hechicería y brujería en la zona en su calidad de Comisario General de las Naciones de Indios. Algunos caciques, entre ellos un cacique de nombre Guenchunao, habían apresado a un indio llamado Juan Pichunante, acusado por voz pública de haber intentado dar muerte a algunos caciques amigos de espańoles. Este será el punto de inicio para el desarrollo de un proceso por brujería que involucrará a casi una docena de indígenas ${ }^{10}$. Entre el conjunto de procesados, figura una india de cuarenta ańos llamada Guenteray. Se trata de la hija del Cacique Quipaina, dueńo de una de las cuevas donde se realizan las "juntas secretas" y es, curiosamente, la única mujer imputada en un delito cuyo referente más cercano -el modelo demonológico tardomedieval- hacía de la mujer una figura especialmente proclive a estas prácticas ${ }^{11}$.

\footnotetext{
9 "Es jeneral abuso en este obispado, no solo en la campaña, sino en las ciudades, atribuir cualquier accidente no conocido por falta de médicos, a maleficio, i a valerse de curanderos machis, con varias supersticiones diabolicas, que usa su ignorancia; i siendo lo refereido gravisimo pecado, asi en el que cura, como en el enfermo, reagravado con la cualidad de reservado en el num 8 cap 12 de ellos; se manda a toda la feligresia, se abstengan de tales curaciones, so cargo de dicha culpa reservada, i que se procederá en lo judicial por todo rigor de derecho". Colección Sínodos Americanos, Sínodo de Concepción (Chile) 1744. Serie dirigida por Horacio Santiago Otero y Antonio García y García, Madrid-Salamanca, CSIC. Universidad Pontificia de Salamanca, 1984, Capítulo XV, Constitución I, pp. 141.

${ }^{10}$ Información levantada por el Capitán Don Antonio de Soto Pedreros, por orden del Presidente Don Tomás Marin de Poveda, contra varios indios acusados de brujos y hechiceros. A.N.S, Manuscritos Medina, T. 323, fojas 1-202. Sala Medina, rollo Ms. M85, Biblioteca Nacional.

${ }^{11}$ No nos referimos únicamente al Malleus Maleficarum de Institoris y Sprenger, cuyo contenido intensamente misógino no deja espacio a duda. La casuística de los procesos, la mantención del estereotipo por vía iconográfica, y manuales propiamente hispanos como las Disquisiciones Mágicas de Martín del Río, retratan a la brujería como una herejía esencialmente femenina, fruto de una larga tradición que habría de comenzar con el canon episcopi. Para una discusión sobre el fenómeno, Ross, Eric. "Syphilis, Misogyny, and Witchcraft in 16th-Century Europe". Current Anthropology, Chicago: The University of Chicago Press, 36/2 (Apr., 1995): 333-337.
} 
De acuerdo a las testificaciones de Juan Pichunante y de un indio conocido como Dunguiguala, las actividades desarrolladas en las cuevas estaban intrínsecamente ligadas a la machi Guenteray. No solo el ingreso a la cueva estaba mediado por sus artes ${ }^{12}$, sino también el castigo de los enemigos de la comunidad. El padre de Pichunante había sido maleficiado por un cacique llamado Naquelpi, y por este motivo planeaba cobrar venganza en el hijo de Naquelpi, por medio de flechassos ${ }^{13}$. El procedimiento es descrito en el proceso: por medio de su aliento, Guenteray permitió que de la mano de Pichunante brotase un "pedasso de canelo en la punta del dedo con quatro ganchos" ", el cual debía ser sumergido en un jarro con "dose axueritos pequeñitos", que según la testificación de Dunguiguala, contenía "agua sacada de la cueva producida de orinas de los Quecubus que son las sabandijas y ficciones que ben del demonio"15. Ya dispuesto con sus instrumentos, Pichunante fue objeto de un nuevo procedimiento, en el que la machi Guenteray nuevamente

${ }^{12}$ Para abrirla hasia un refregado de yervas con agua y entre medio de las seremonias de un canto diabolico salpica con el agua, por manos de la machi Guenteray se forma a un remolino [...] que yba descomponiendo la mata de coiron y assi que la vian que estava desarraigada la levantavan a un ladito de la puerta y entraban por puerta franca para el sentro de la tierra" (Ms. M85, foja 5).

${ }^{13}$ A partir de la obra del jesuita Andrés Febres contamos con algunas noticias sobre esta práctica. En su Arte de la Lengua General, Febres identifica dos voces mapudungun para esta práctica: Pulquitun y Coghtun. La primera significa "Flechar o tirar flechazos los bruxos", y estaría estructurada en torno a la voz Pulqui cuyo significado literal es "flecha". La segunda voz, Coghtun, es traducida por Febres como "dar flechazo los bruxos para hacer daño", donde la partícula Cogh haría referencia a "unos palitos que dan a los españoles para que cuenten los valientes de su nación". Si revisamos las entradas en español para "flechar", la voz escogida es pulquitun, pero con una segunda acepción: "coger flecha o consentir alzamiento". Por su parte, "Flechar los brujos" es traducido como Coghtun y, alternativamente, Cúllin. Febres, Andrés. "Arte de la Lengua General del Reyno de Chile". Lima: Calle de la Encarnación, 1765. (Edición Facsimilar.). Fols. 607 y 457.

${ }^{14}$ A.N.S. Sala Medina. Vol. 304, Rollo Ms. M85, foja 39. De acuerdo a la testificación, un indio de nombre Lemullanca había sido quien en una ocasión introdujo la semilla en su palma, la que Guenteray solo había "llamado". Resulta interesante observar que el Foye o Canelo (Drimys winterii) no es utilizado únicamente en contextos rituales de uso positivo -largamente documentados en los trabajos de campo- sino también constituye un elemento central en la confección de artefactos del maleficio, sirviendo como material base para la fabricación de las llamadas "varillas de encanto" o sencillamente "canutos" y que conforman el soporte material de los "flechassos" por medio de los cuales se inocula el mal.

${ }^{15}$ A.N.S. Sala Medina. Vol. 304, Rollo Ms. M85, foja 6. En el proceso de Chillán 1749, las "aguas de sabandijas que tenían recojidas en un jarro" permitían a los maleficiadores cambiar de forma a zorros, perros o pájaros. Casanova Guarda, Holdenis. "Diablos, brujos y espíritus maléficos: Chillán, un proceso judicial del siglo XVIII”. Temuco: Eds. Univ. de La Frontera, (1994): 171. La voz "Quecubus" o derechamente "Hecubos" corresponden a la transcripción de la voz indígena "Wekufe". La similitud fonética de Hecubos con íncubos y súcubos de la tradición demonológica europea (pormenorizadamente abordado por el Malleus Maleficarum) no es mera coincidencia. Para los jueces dichos referentes forman parte de las matrices de comprensión de la brujería. 
tuvo participación. Reunida junto a Lemullanca y Dunguiguala, Guenteray había "adobado con distintas yerbas" a Juan Pichunante para que "fuesse a dar el flechasso bolando en una figura de pajaro a modo de condor". Gracias al theriomorfismo, el homicidio del hijo de Naquelpi podía ser hecho en total impunidad: en forma de cóndor, ningún perro le ladraría ni ningún hombre le podría identificar ${ }^{16}$. No fue esta la única vez en que la machi Guenteray colaboró con un maleficio. Prácticamente con las mismas operatorias rituales Pichunante dio muerte al machi Calbupan, a solicitud de la propia Guenteray, "por sentimiento que thenia de que no la avia brindado con chicha en un baile que hubo de bruxos"17.

En el contexto del juicio, Guenteray era una maleficiadora. Mientras sus conocimientos sobre el uso de hierbas -tanto para la elaboración de flechassos como para inducir la metamorfosis animal- permitían que fuese sindicada como hechicera, su activo rol en las "juntas secretas" celebradas en las cuevas era una prueba inequívoca de su ejercicio de la brujería. Pese a estos antecedentes, a ser identificada como una especialista y a ser la única mujer de esta sociedad clandestina, ninguno de estos elementos supuso un agravante ni implicó el inicio de pesquisas independientes al caso.

Una clave de lectura puede estar dada en la defensa que el coadjutor de indios, Don Gerónimo Agustín de la Vega, realizó para solicitar la absolución de Guenteray. Junto a los tópicos generales del abandono espiritual en el que estaban los indios, el defensor señala que estos han vivido apartados de la luz misional, "unos creiendo en las supersticiones de el demonio y otros en la virtud de las yerbas por via de medicamento para la salud corporal"18. En la defensa de Guenteray, señala que "no deve padezer la calumnia de bruxa ni ser molesta por esta caussa". Es llamada "arbolaria", idea que ocasionalmente se desliza en la propia redacción de la justicia, para remitir su universo de prácticas estrictamente a la manipulación de hierbas con fines curativos.

El caso del indio Joseph Acosta nos entrega sorprendentes antecedentes en esta dirección. Apresado ilegítimamente un 8 de junio de 1739 en la ciudad de Santiago, Joseph fue conducido a casa de un vecino de la Calle Santa Clara, don Miguel Zańartu quien "vio que tenía una de las manos una bolsa colorada". Este es el inicio de un proceso motivado por el contenido de dicha bolsa, el cual contenía "varias inmundicias que le causaron temor como que denotavan ser de echiserias" ${ }^{19}$. La justicia infirió que por

\footnotetext{
${ }^{16}$ A.N.S. Sala Medina. Vol. 304, Rollo Ms. M85, Foja 10.

${ }^{17}$ A.N.S. Sala Medina. Vol. 304, Rollo Ms. M85, Foja 40.

${ }^{18}$ A.N.S. Sala Medina. Vol. 304, Rollo Ms. M85, Foja 97.

${ }^{19}$ A.N.S. R.A Vol. 1759, Pieza 20. Foja 1. Laura de Mello e Souza, en su estudio de la hechicería y religiosidad popular en Brasil colonial constata también la presencia de estas bolsas, las que reciben el nombre de "Bolsas de Mandinga". Compartimos la opinión de la autora, la cual señala: "Las bolsas son tal vez la más sincrética de todas las prácticas mágicas y de hechicería conocidas entre nosotros:
} 
estos antecedentes, debía tratarse de "echisero o brujo", practicante de "maleficios o supersticiones". A diferencia del caso anterior, el proceso se inició sin existir causa criminal alguna. En estricto rigor, los dos delitos imputados fueron portar una bolsita roja de contenido indeterminado, y un relicario cristiano con una inscripción en papel. Ninguno de estos antecedentes justificaban un cargo criminal y, sin embargo, bastaron para su encarcelamiento preventivo. Es posible que este celo se viese catalizado por la presencia de la reliquia, que abre la puerta a un posible uso sacrílego ${ }^{20}$. Tras varias testificaciones, el proceso llega a un punto muerto. El indio Joseph de Acosta negó todos los cargos que se le imputaban y, por sobre todo, negó que el contenido de la bolsita pudiese ser la causante de algún eventual maleficio.

Para determinar la peligrosidad del hechizo que portaba el indio Joseph fue convocado un especialista. A diferencia del proceso a Juana Codosero en

son la resolución especifica de habitos culturales europeos, africanos e indígenas; congregan al tradición europea de los amuletos con el fetichismo amerindio y las costumbres de las poblaciones de Africa". Las bolsas analizadas por la autora son blancas. Mello e Souza, Laura de. El diablo en la tierra de santa cruz: hechicería y religiosidad popular en el Brasil colonial. Madrid: Alianza Editorial, (1993): 193. También el cura Alonso de Peña menciona la presencia de estos dispositivos mágicos en su Itinerario para Parrocos, y relata la historia de un cura doctrinero que había conocido, el cual había perseguido a un famoso hechicero, apresándolo, destruyendo una bolsita que contenía "bierbas y otras porquerias" y haciendo auto público para ejemplificar la gravedad del delito. Los indios del sector se compadecieron del cura pensando que con toda seguridad el hechicero le mataría. "Tome ocasion para darles a entender el inmenso poder de Dios, y lo poco o nada que el demonio puede con sus minstros". De la Peña, Alonso: "Itinerario para Parrocos de Indios. En que se tratan las materias mas particulares tocantes a ellos para su buena Administracion". Quito: Anuario Histórico Jurídico Ecuatoriano IX, 1985. Lib. II, Trat. V. Secc. I. El mismo Alonso de la Peńa remite el caso de un cura doctrinero de Quito, quien al momento de apresar a un a un famoso hechicero de la zona, encontró en posesión de este una bolsita que contenía "bierbas y otras porquerias". El proceso terminó con un auto público para ejemplificar la gravedad del delito, a la vista de los indios del sector, quienes se compadecieron del cura doctrinero, seguros que el hechicero cobraría venganza. El cura no perdió la oportunidad "para darles a entender el inmenso poder de Dios, y lo poco o nada que el demonio puede con sus ministros". Lib. II, Trat. V. Secc. I.

${ }^{20}$ Hacemos hincapié en que la visión de estas prácticas se realiza desde el área de conocimiento de los jueces locales, y no de la justicia eclesiástica. El juez cuestionó la presencia de estos papelitos en el relicario, exigiéndole al reo que dé explicaciones sobre su porte cuando, recordemos, el propio indio Joseph "dio a su voluntad un relicario que contenia cierta orasión diziendo leyese aquel papel". La tenencia de la "reliquia" por parte de Joseph podría remitir a dos posibilidades. La primera y más evidente es el robo de objetos sagrados, anatematizado en las disposiciones sinodales de 1626 por el peligro de "alguna indecencia y que usen los indios del óleo y crisma para algunos maleficios". En: "Sínodo Diocesano de Santiago de Chile celebrado en 1626, Transcripción, introducción y notas de Fr. Carlos Oviedo Cavada...." Cap. I, Const. VIII. La segunda posibilidad proviene de una pista que encontramos en una de las Cartas Annuas Jesuitas. Se señala: "Es costumbre en los jesuitas, repartir hojitas con el nombre de un Santo, cuya fiesta se celebra en el mes que comienza, para venerarlo durante el mes que corre. Las Cartas Anuas mencionan varias veces esta costumbre, que era muy popular entre los indios cristianos". Documentos para la historia de Argentina... ibid, p. 434. 
1693, no fue un médico el convocado ${ }^{21}$. Ni siquiera un español. En contra de los lineamientos dispuestos por la legislación eclesiástica, el perito fue precisamente un machi el llamado de nombre Juan de León, cacique del pueblo de Lluquen y "persona que notoriamente cura enfermedades de maleficio", será convocado para deliberar sobre el contenido de la bolsita ${ }^{22}$. Su respuesta será taxativa: "son dichas porquerias para hacer maleficios y no para curarlos" 23 .

¿Cómo se explica que un machi -figura central de los ritos supersticiosos locales- haya sido convocado por la Justicia Real para actuar como especialista? ¿Cómo se explica a la luz de las disposiciones sinodales, que penaban con excomunión y azotes toda asociación con estas figuras? La información que Ana Mariella Bacigalupo nos presenta sobre la imagen del mundo colonial hispano frente a la Machi no hace sino acentuar la extrañeza de estos vínculos. La autora, fundamentalmente a partir del estudio de crónicas, sintetiza la visión hispana de los machi hombres (weye) bajo los apelativos de "putos" o "sodomitas", y el estereotipo que de su imagen puede verse en la descripción que hace Núñez de Pineda y Bascuñán resulta esclarecedora: "llegó un indio de tan mal figura, que su traje, perverso rostro y talle [...] parecía un Lucifer en sus facciones, talle y traje", mientras el padre Luis de Valdivia, traduce el término weye como "sodomita" y weyun como "pecado nefando" 24.

No solo la india Guenteray no es procesada como hechicera o bruja, pese a tener cargos de homicidio por vías mágicas, sino incluso un machi weye como el cacique don Juan de León es llamado por la Justicia Real para testificar en calidad de especialista, de forma homologable a la que el Licenciado Joseph Davalos,

\footnotetext{
${ }^{21}$ Se trata del licenciado Joseph Davalos y Peralta, médico de la ciudad de Santiago, convocado por la justicia para realizar peritajes en torno a la muerte del capitán Juan Gutiérrez. El proceso se encuentra en: A.N.S. Fondo Real Audiencia, Vol. 2529, pieza 2. En tanto causa de hechicería, es analizado en: Valenzuela, Eduardo: "Maleficio...". p. 63.

22 Sinodo diocesano de Santiago de Chile. Celebrado en 1626 por el ilustrísimo señor Francisco Gonzalez de Salcedo. Transcripción, introducción y notas de Carlos Cavada Oviedo, "Historia", No3, Santiago 1964. 313-360 (Cap. III, Const. III, p. 336). El sínodo no prohíbe explícitamente, por cierto, convocar a machis como peritos judiciales, sino vincularse a ellos en cuestiones relacionadas con la sanación. Mencionamos la constitución sinodal para señalar que la esfera de conocimiento de la machi, anatematizada en la legislación eclesiástica, posee por el contrario un espacio de validez dentro de la justicia ordinaria. Más aún, si Juan de León era persona que notoriamente curaba enfermedades de maleficio es porque tenía una clientela bastante amplia, incluyendo al gobernador Thomas de la Sierra, quien había acudido a él por tener un hijo enfermo. A.N.S Fondo Real Audiencia Vol. 1759, Pieza 20. Foja 4R.

${ }^{23}$ A.N.S Fondo Real Audiencia Vol. 1759, Pieza 20. Foja 4R.

${ }^{24}$ Bacigalupo, Ana Mariella. "La lucha por la masculinidad del machi: políticas coloniales de género, sexualidad y poder en el sur de Chile". Santiago de Chile: Revista de Historia Indígena, No 6. Departamento de Ciencias Históricas, Universidad de Chile (2002): 39.
} 
médico de la ciudad de Santiago, hiciera para determinar si los sesos de asno constituían la razón de la muerte del Capitán Gutiérrez. Aunque nos podríamos sentir tentados a pensar que fue la calidad de "cacique" lo que permitió a Juan de León gozar de ciertas prebendas por parte de la justicia, hay casos que nos permiten contrastar esta situación. En 1710, Tomasa Briceño, cacica de Malloa, perdió la custodia de su nieta Petrona en manos de don Antonio Garses, "atendiendo a la mejor criansa y educasion" de la niña, ya que siendo "preguntada la dicha India Petrona sobre los prinsipales misterios de Nuestra sancta fee catholica se allara sin conoserlos $^{25}$. Garses cierra toda posibilidad de reencuentro entre abuela y nieta, al recordar que Tomasa habría sido anteriormente "procesada por bruja". De manera especialmente significativa, la justicia omitió en todo momento el tratamiento de "cacica", llamándola sencillamente "yndia Tomasa", mientras el defensor licenciado Francisco Ruiz reivindicó su calidad tantas veces como fue necesario. A diferencia de Juan de León, Tomasa no tuvo un trato distintivo por su calidad de cacica, título que los querellantes omitieron a lo largo de todo el proceso. A diferencia del Cacique de Lluquen, Tomasa no era reconocida como machi.

La dicotomía existente entre la visión negativa que figura en las crónicas y los sínodos, y aquella que se expresa en las fuentes judiciales que hemos analizado, nos obliga a repensar el vínculo de estos agentes en la sociedad colonial. Podemos hablar de instrumentalización cuando analizamos la convocatoria del machi weye Juan de León al proceso del indio Joseph y lo confrontamos a la realidad de la cacica Tomasa Briceńo, en el primer caso para inculpar al reo, en el segundo, para negar la solicitud de restitución de la indiecita Petrona a su abuela. Sin embargo, la laxitud que la justicia manifiesta frente a la machi Guenteray en el proceso de Concepción 1693 o al machi weye Pedro Chipayllen en el proceso de Chillán $1749^{26}$, nos obliga a matizar la idea de un vínculo inmediato entre machi y bruja: dicha asociación no fue compartida por todo el mundo hispano, ni tampoco fueron sus capacidades curativas el motor de dicha imputación. Intentaremos ahondar más en esta propuesta.

En su estudio del delito de hechicería en el derecho indiano, Dougnac nos recuerda que las fuentes de información a la que los jueces podían echar mano fueron esencialmente las Leyes de Indias, las cuales se nutrían esencialmente de

\footnotetext{
${ }^{25}$ A.N.S. Fondo Real Audiencia Vol. 2576, p. 3, Foja 23. Este proceso es abordado también en: Pfenniger, Catalina. "Ruralización y Fe: Desestructuración material y espiritual de los indígenas de la doctrina de Malloa (1635-1744)". Tesis de Licenciatura en Historia. Santiago de Chile: Pontificia Universidad Católica de Chile, 2002.

${ }^{26}$ El nombre de este machi, mencionado al inicio del proceso contra los brujos de Chillán, desaparece con el correr del proceso, y no fue blanco de indagatorias especiales por parte de la justicia. Para una revisión pormenorizada de este proceso, ver: Casanova Guarda, Holdenis. Diablos, brujos y espiritus maléficos: Chillán, un proceso judicial del siglo XVIII. Temuco: Eds. Univ. de La Frontera, 1994.
} 
las Siete Partidas ${ }^{27}$. Las noticias sobre prácticas heterodoxas son, sin embargo, más bien escuetas. El código alfonsí identifica a un primer tipo de sujeto -y de práctica- en los ya mencionados adivinos, distinguiendo aquellos que logran sus fines lícitamente a través de "arte de astronomía" de los otros: agoreros y sorteros que consultan objetos o animales para vaticinar el futuro ${ }^{28}$. Apartándose de la superstitio, el código menciona también la hechicería y nigromancia como ciencias "para encantar a los demonios" y lograr con ello fines ilícitos tales como encantar hombres y mujeres -ligaduras- o encontrar "cosas temporales que codician" ${ }^{29}$. Las prácticas de ambos grupos son consideradas indistintamente pecados o delitos, salvo una excepción. En el Título XXIII, Ley 3ra, el código señala:

Los que hizieren encantamientos o otras cosas con entencion buena asi como sacar demonios de los cuerpos de los onbres o para deflegar a los que fuesen marido o muger que no pudiesen convenir o para delatar nuve que traxiese granizo o niebla porque no conociese los frutos o para matar langosta o pulgo que dańa el pan o el vino o por alguna otra razon o echo semejante destas no debe aver pena antes dezimos han que de recebir galardon por ello ${ }^{30}$.

Estas "buenas razones" son precisamente las que el Coadjutor de Indios Gerónimo Agustín de la Vega utilizará para solicitar la absolución de la machi Guenteray. Para el defensor, los indígenas acusados de brujería en el proceso de Concepción 1693 simplemente habían vivido apartados de la luz misional, "unos creiendo en las supersticiones de el demonio y otros en la virtud de las yerbas por via de medicamento para la salud corporal"31. Desde su óptica, Guenteray "no deve padezer la calumnia de bruxa ni ser molesta por esta caussa". Es, por el contrario, una "arbolaria", idea que ocasionalmente se desliza en la propia redacción de la justicia, para remitir su universo de prácticas estrictamente a la manipulación de hierbas con fines curativos ${ }^{32}$.

\footnotetext{
${ }^{27}$ Ver nota 3.

${ }^{28}$ Las Siete Partidas que mando collegir el Catholico Rey Don Alfonso el nono, Rey de Castilla y León. Edic. Paulo de Colonia e Johánnes Pegniczer de Nuberga e Magno e Thomas, 24 de diciembre de 1491. Título XXIII, Ley 3ra. Edición facsimilar en: Sala Medina SM 390. Partida VII, Título XXIII, Ley 1 ra.

${ }^{29}$ Ibid, Partida VII, Título XXIII, Ley 2 da.

${ }^{30}$ Ibid, Partida VII, Título XXIII, Ley 3ra.

${ }^{31}$ Ms M85, Foja 97.

${ }^{32}$ A.N.S. Sala Medina. Vol. 304, Rollo Ms. M85, Foja 3. Encontramos también algunas reflexiones en esta dirección en la obra de Ovalle: "Ay muchas yeruas muy medicinales, y de grandes virtudes conocidas solamente de los Indios que llaman machis, que son sus medicos, las quales las occultan particularmente de los Españoles, a quienes por grande amistad comunican la virtud de una, ó otra, reseuando para si la sciencia de las demas, la qual passa solo de padres a hijos; $y$ son estos medicos o machis muy estimados assi de los indios como tambien de los mesmos Españolos, que los llaman en el maior aprieto de sus enfermedades, y experimentan admirables curas". Ovalle, Alonso de. Historica relacion
} 
El peso de la jurisdicción del código alfonsí se ve reflejado incluso en textos eclesiásticos como el Itinerario para Párrocos de Indios (1668), escrito por Alonso de Peña Montenegro, y de enorme difusión en el contexto americano ${ }^{33}$. Para precaver a los párrocos de sus obligaciones, esto es, hacerse cargo de la porción que les corresponde en la punición de este delito de "fuero mixto":

Aunque hay en el Codigo algunos textos, y otros en las Partidas, que excusan a los hechiceros, que con buena Fe usan de sus hechizos [...] estos textos los entienden los Doctores, en quanto a las penas del fuero exterior, pero no en cuanto al pecado y fuero interior, que tanto aborrece este abominable pecado ${ }^{34}$.

Las machi, en tanto sanadoras, no solo quedan fuera de la jurisdicción de los tribunales ordinarios, fuera de sus áreas de competencia e incluso de sus intereses, sino que su propia naturaleza parece contravenir los lineamientos propios de la matrices conceptuales que en otras condiciones determinarían su persecutoria criminal civil. Aunque son capaces de articular plantas, animales y otras sustancias en artefactos con virtudes ocultas - hechicería-, e incluso causar con estos la enfermedad o muerte -maleficio- de un tercero, lo cierto es que no se procede contra ellas. Aunque se describe su importancia central en los ritos que la comunidad celebra en cuevas, con adoración explícita de entidades demoníacas -brujería-, nuevamente no se les exime de una responsabilidad mayor. Todo esto considerando, valga la pena recordarlo, que estas mujeres reúnen los atributos centrales del modelo de la brujería tardo-medieval, una de las matrices fundamentales en base a las cuales la justicia civil procederá3 ${ }^{35}$. Pese a todo esto, no se convirtió en el objeto persecutorio de la justicia, ni se desplegó sobre ellas los instrumentales usados contra otros reos. Quizás, en el terreno de las prácticas, todo pasa a un segundo plano en una sociedad colonial que demanda de forma apremiante sus saberes, como lo reconocerá dolorosamente el Sínodo de Concepción $1744^{36}$.

del reyno de Chile y de las misiones y ministerios que exercita en el la Compañia de Jesus a nuestro señor Jesu Christo dios hombre y á la santissima virgen y madre Maria señora del cielo, y de la tierra y á los santos Joseph, Joachin, Ana sus padres y abuelos. Rome: Caballo, 1646. (Edición facsimilar), pp. 5-6.

${ }^{33}$ Se trata del proceso de Chillán 1749, donde el cura Simón de Mandiola cita la obra de Alonso de Peńa para intentar probar su jurisdicción como juez eclesiástico en causas cuya competencia correspondía a la Justicia Real, sin éxito.

${ }^{34}$ De la Peña, Alonso. “Itinerario para Parrocos...” Lib. II, Trat. V. Secc. VI. En: Anuario Histórico Jurídico Ecuatoriano IX, Quito, 1985. Lib. II, Trat. V. Prólogo. Ofrece como soluciones alternativas y piadosas encomendarse a Dios, solicitar los Exorcismos, la invocación de Jesucristo, el ayuno y el uso y porte de signos sagrados.

${ }^{35}$ Valenzuela, Eduardo: "Maleficio...". p. 28.

${ }^{36}$ Ver nota 2. 
Las continuas disposiciones sinodales para anatematizar toda asociación con las machi, dispuestas en el Sínodo de 1626, refrendadas en el Sínodo de $1688^{37}$, reafirmadas en el Sínodo de Concepción $1744^{38}$ y mantenidas en vigencia hasta entrado el s. XIX ${ }^{39}$, son el ejemplo inequívoco del vigor del vínculo de las machi con un segmento importante de la sociedad colonial chilena que, al igual que el gobernador Thomas de la Sierra, validará la efectividad de sus medios y acudirá en pos de su conocimiento, en los intersticios de la legalidad. El estatuto de la machi en los procesos por maleficio dependerá, siempre, del sujeto que enuncia las preguntas, y del contexto que exige su respuesta.

\section{REFERENCIAS}

Bacigalupo, Ana Mariella. "La lucha por la masculinidad del machi: Políticas coloniales de género, sexualidad y poder en el sur de Chile", Revista de Historia Indígena, No 6. Departamento de Ciencias Históricas, Universidad de Chile, (2002): 29-64.

Casanova Guarda, Holdenis. Diablos, brujos y espiritus maléficos: Chillán, un proceso judicial del siglo XVIII. Temuco: Eds. Univ. de La Frontera, 1994.

Dougnac, Antonio. "El delito de hechicería en Chile Indiano", Revista Chilena de Historia del Derecho 8 (1981): 93-107.

García Gallo, Alfonso."Metodología de la historia del derecho indiano". Publicaciones del Seminario de Historia y Filosofía del Derecho de la Facultad de Ciencias Jurídicas y Sociales de la Universidad de Chile, 1970.

Ginzburg, Carlo. Historia Nocturna, un desciframiento del aquelarre. Barcelona: Munchkin Editores, 1991.

\footnotetext{
${ }^{37}$ Sínodos de Santiago de Chile de 1688 y 1763 , Bernardo Carrasco Saavedra, Manuel de Alday y Aspee, Madrid: Instituto Francisco Suárez del Consejo Superior de Investigaciones Científicas, (1983): 71.

${ }^{38}$ Colección Sínodos Americanos, Sínodo de Concepción (Chile) 1744. Serie dirigida por Horacio Santiago Otero y Antonio García y García, Madrid-Salamanca, CSIC. Universidad Pontificia de Salamanca, 1984.

${ }^{39}$ Iglesia Católica. Diócesis de Ancud (Chile). El primer sinodo chileno de la época republicana: Ancud 1851. Ed. Fernando Retamal. Santiago de Chile: Ediciones Universidad Católica de Chile, Santiago, 1983. Título XX. Constitución VIII, p. 171.
} 
Giordano, Oronzo. Religiosidad popular en la Alta Edad Media. Madrid: Gredos, 1983.

Mello e Souza, Laura de. El diablo en la tierra de santa cruz: Hechicería y religiosidad popular en el Brasil colonial. Madrid: Alianza Editorial, 1993.

Ots y Capdequi, José María. Manual de historia del derecho español en las Indias y del derecho propiamente indiano. Buenos Aires: Losada, 1945.

Pérez González, María Luisa. "Los caminos reales de América en la legislación y en la historia", Anuario de Estudios Americanos 58/1 (2001).

Pfenniger, Catalina. "Ruralización y Fe: Desestructuración material y espiritual de los indígenas de la doctrina de Malloa (1635-1744)". Tesis de Licenciatura en Historia. Santiago, Chile: Pontificia Universidad Católica de Chile, 2002.

Ross, Eric. "Syphilis, Misogyny, and Witchcraft in 16th-Century Europe." Current Anthropology, Chicago: The University of Chicago Press, 36/2 (April, 1995): 333-337.

Serrano Sanz, Rosa. "Hacia un nuevo planteamiento del conflicto paganismocristianismo en la Península ibérica", Revista 'Ilu, Madrid, 1995.

Valenzuela, Eduardo. Maleficio. Historias de hechiceria y brujería en Chile colonial. Santiago, Chile: Pehuén Editores, 2013.

Valenzuela, Eduardo. "Vino nuevo en odres viejos: Superstitio et idololatria en la Espańa alto-medieval”. En José Manuel Cerda (Ed.), El mundo medieval: Legado y Alteridad. Santiago, Chile: Editorial Finis Terrae, 2009.

\section{Fuentes publicadas}

Colección Sinodos Americanos, Sinodo de Concepción (Chile) 1744. Serie dirigida por Horacio Santiago Otero y Antonio García y García, Madrid-Salamanca, CSIC. Universidad Pontificia de Salamanca, 1984.

De la Peña, Alonso. "Itinerario para Parrocos de Indios. En que se tratan las materias mas particulares tocantes a ellos para su buena Administracion". Quito: Anuario Histórico Jurídico Ecuatoriano IX, 1985. 
Diccionario de la lengua castellana en que se explica el verdadero sentido de las voces, su naturaleza y calidad con las phrases o modos de hablar, los proverbios o refranes, y otras cosas convenientes al uso de la lengua. Tomo IV. Madrid: En la Impr. de Francisco del Hierro, 1726-1739. 6 v.(Edic. Facsimilar).

Documentos para la Historia de Argentina. Tomo XIX. "Cartas anuas de la provincia del Paraguay, Chile y Tucumán, de la Compañía de Jesús 1609 -1614”. Con advertencia de Emilio Ravignani e introducción del P. Carlos Leonhardt, Buenos Aires, Instituto de Investigaciones Históricas, Facultad de Filosofía y Letras, Peuser, 1927.

Febres, Andrés. "Arte de la Lengua General del Reyno de Chile". Lima: Calle de la Encarnación, 1765. (Edición Facsimilar.).

Iglesia Católica. Diócesis de Ancud (Chile). El primer sinodo chileno de la época republicana: Ancud 1851. Ed. Fernando Retamal. Santiago, Chile: Ediciones Universidad Católica de Chile, 1983.

Las Siete Partidas que mando collegir el Catholico Rey Don Alfonso el nono, Rey de Castilla y León. Edic. Paulo de Colonia e Johánnes Pegniczer de Nuberga e Magno e Thomas, 24 de diciembre 1491.

Ovalle, Alonso de. Historica relacion del reyno de Chile y de las misiones y ministerios que exercita en el la Compañía de Jesus a nuestro señor Jesu Christo dios hombre y á la santissima virgen y madre Maria señora del cielo, y de la tierra y á los santos Joseph, Joachin, Ana sus padres y abuelos. Rome: Caballo, 1646. (edición facsimilar).

Sinodo Diocesano de Santiago de Chile celebrado en 1626, por el ilustrísimo señor Francisco González de Salcedo. Transcripción y notas por Oviedo Cavada, Carlos, Revista Historia, (3): 313-360, Santiago, 1964.

Sinodos de Santiago de Chile de 1688 y 1763, Bernardo Carrasco Saavedra, Manuel de Alday y Aspee, Madrid: Instituto Francisco Suárez del Consejo Superior de Investigaciones Científicas, 1983.

Valdivia, Luis de. Sermón en lengua de Chile. De los mysterios de nuestra santa fe catholica, para predicarla a los indios infieles del reyno de Chile, dividido en nueve partes pequeñas, acomodadas a su capacidad. Santiago, Chile: Elzeviriana, 1897. 